\title{
O ESTADO DA ARTE DAS PESQUISAS EM DISSERTAÇÕES DE MESTRADO EM ENSINO DE GEOGRAFIA: CONTRIBUIÇÃO PARA UMA AVALIAÇÃO A PARTIR DAS PESQUISAS NOS PROGRAMAS NACIONAIS DE STRICTO SENSU (2008-2012)
}

\author{
State of the art of research thesis masters in teaching of \\ geography: an evaluation from the research in the national \\ stricto sensu (2008 - 2012)
}

Estado del arte de masters tesis de investigación en enseñanza de la geografía: una evaluación de la investigación en el nacional sensu stricto (2008 - 2012)

Ricardo Lopes Fonseca

Doutorando pelo Programa de Pós-Graduação em Geografia na Universidade Estadual de Londrina (UEL). E.mail: ricardolopesfonseca@hotmail.com

\section{Rosana Figueiredo Salvi}

Profa. Dra . do Programa de Pós Graduação em Geografia da Universidade Estadual de Londrina. Departamento de Geociências.E.mail: ro06salvi@gmail.com

\section{Eloiza Cristiane Torres}

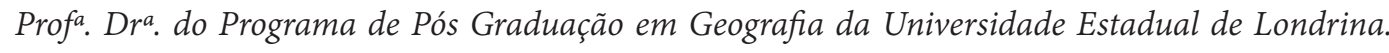
Departamento de Geociências.E.mail: elotorres@hotmail.com

\section{Resumo}

Neste artigo será apresentado o Estado da Arte das pesquisas em Ensino de Geografia a partir da análise das Dissertações de mestrado defendidas no Brasil, entre os anos de 2008 e 2012, verificadas no banco de teses da CAPES. O objetivo é evidenciar os temas pesquisados sobre o Ensino de Geografia, bem como apresentar as instituições de ensino superior que incentivam este tipo de pesquisa no Brasil. As tabelas, gráficos e mapas estão inseridos para proporcionar ao leitor uma noção sobre os temas das pesquisas produzidas e sua espacialização no território nacional. Encontrou-se 257 Dissertações defendidas que tratam sobre o Ensino de Geografia, nos mais diversos programas de pós-graduação Stricto Sensu. Destacam-se os seguintes temas: sobre a escola, políticas públicas, modalidade de educação, sobre o professor de Geografia, conteúdos geográficos e recursos didáticos. Sobressaem os assuntos relacionados aos recursos didáticos, aos conteúdos de Geografia e à formação docente. No tocante à distribuição geográfica, a produção das pesquisas em Ensino de Geografia concentra-se na região sudeste e sul. Por fim, por ser uma atividade árdua e complexa, o Estado da 
Arte possui caráter crítico e reflexivo, por isso, auxilia no desenvolvimento de novas demandas de pesquisa, conceitos e paradigmas.

Palavras-chave: Estado da Arte; Ensino de Geografia; Produção Acadêmica; Stricto Sensu.

\section{Abstract}

This paper deals with the state of the art research in Teaching Geography from the analysis of master Dissertations in Brazil, between the years 2008 and 2012, recorded in the CAPES thesis database will be presented. The aim is to highlight the topics searched on the Teaching of Geography and present institutions of higher education that encourage such research in Brazil. The tables, graphs and maps are included to give the reader a sense of the themes of the research produced and spatialization in the country. Met 257 Dissertations dealing on the Teaching of Geography in many different programs post- graduate studies. Stand out in the following subjects: about school, public policy, education mode on the teacher of Geography, Geographical content and teaching resources. Stand the issues related to teaching, the content of Geography and teacher training resources. Regarding geographical distribution, the production of research focuses on the on the southern and southeastern. Finally, as a complex and strenuous activity, the state of the art has a critical character and reflexive, so it helps to develop new research demands, concepts and paradigms.

Keywords: State of the Art; Teaching Geography; Academic Production; Stricto Sensu.

\section{Resumen}

En este artículo se presenta el estado del arte de la investigación en la enseñanza de la Geografía a partir del análisis de las disertaciones magistrales en Brasil, entre los años 2008 y 2012, registrados en la base de datos de tesis CAPES. El objetivo es poner de relieve los temas investigados en la Enseñanza de la Geografía y de las instituciones actuales de la educación superior que fomenten este tipo de investigación en Brasil. Las tablas, gráficos y mapas se incluyen para dar al lector una idea acerca de los temas de la investigación producidos y su distribución espacial en el territorio nacional. Met 257 Disertaciones tratan sobre la Enseñanza de la Geografía en los distintos programas de estudios de postgrado. Se destacan en las siguientes materias: sobre la escuela, la política pública, el modo de la educación en el profesor de Geografía, contenido geográfico y recursos didácticos. Párese las cuestiones relacionadas con la enseñanza, el contenido de la Geografía y de los recursos de formación del profesorado. En cuanto a la distribución geográfica, la producción de la investigación en la enseñanza de la Geografía se centra en el Sur y Sureste. Por último, al ser una actividad difícil y compleja, el estado de la técnica presentan un carácter crítico y reflexivo, lo que ayuda a desarrollar nuevas demandas de investigación, conceptos y paradigmas.

Palabras clave: Estado del Arte; Geografía Enseñanza; Producción Académica; stricto sensu. 


\section{INTRODUÇÃO}

Nesta investigação serão apresentados dados sobre a situação das pesquisas em Ensino de Geografia a partir de análises da quantidade da produção acadêmica, referente as Dissertações defendidas, compreendendo o período entre os anos de 2008 e 2012.

A partir da apresentação de tabelas, gráficos e mapas, o leitor poderá obter conhecimento acerca dos temas e assuntos de pesquisas que estão sendo realizadas nas universidades sobre o Ensino de Geografia, bem como em quais programas de Stricto Sensu tem sido realizadas tais pesquisas.

A espacialização destes resultados, em território nacional, é apresentada com a finalidade de evidenciar onde estão os centros de excelência em pesquisas em Ensino de Geografia no Brasil; desta forma o leitor que possui interesse por esta temática, e que deseja trabalhar com esse assunto poderá, através desta pesquisa, saber, por exemplo, onde pode estar localizada a instituição de ensino superior que dará suporte e apoio às suas pesquisas.

\section{Importância do Estado da Arte para a Pesquisa Acadêmica}

As pesquisas denominadas "Estado da Arte" (EAr) a partir do caráter bibliográfico, pelo qual é definido, de forma geral, possuem uma ideia em comum: mapear a produção acadêmica nas mais diversas áreas do conhecimento. Após esse mapeamento abre-se, naturalmente, o debate acerca do tema levantado, com o objetivo de responder, de acordo com Ferreira (2002, p. 258),

[...] que aspectos e dimensões vêm sendo destacados e privilegiados em diferentes épocas e lugares, de que formas e em que condições têm sido produzidas certas dissertações de mestrado, teses de doutorado, publicações em periódicos e comunicações em anais de congresso e de seminários.

O EAr também é conhecido por sua notoriedade em realizar um método cujas características se mostrem como algo inventariante e descritivo em relação à produção acadêmica e científica acerca do tema que procura pesquisar, "[...] à luz de categorias e facetas que se caracterizam enquanto tais em cada trabalho e no conjunto deles, sob os quais o fenômeno passa a ser analisado". (FERREIRA, 2002, p. 258).

O EAr refere-se a uma atividade árdua e complexa, justamente por seu caráter crítico e reflexivo. Auxilia na otimização e também no desenvolvimento de novas demandas de pesquisa, conceitos e paradigmas. 
Haddad (2002, p. 09) conceitua o EAr como sendo os estudos que:

[...] permitem, num recorte temporal definido, sistematizar um determinado campo de conhecimento, reconhecer os principais resultados da investigação, identificar temáticas e abordagens dominantes e emergentes, bem como lacunas e campos inexplorados abertos a pesquisas futuras.

Um dos objetivos principais deste tipo de pesquisa é buscar compreender o conhecimento que existe sobre algum tema, a partir de um recorte temporal específico, além de sua sistematização e análise acerca do assunto, que não somente resgata como também condensa a produção acadêmica num campo específico.

Este tipo de pesquisa é de cunho bibliográfico, pois há uma sistematização quanto ao modo e às condições da produção acadêmica (artigos publicados em periódicos, Dissertações e Teses defendidas em programas de Stricto Sensu e artigos publicados em anais de congressos). Logo, o EAr é uma pesquisa bibliográfica, sistemática, analítica e, acima de tudo, crítica sobre a produção acadêmica acerca de algum tema específico.

Normalmente, as pesquisas desse cunho baseiam-se nas leituras dos resumos. Entretanto, Barreto e Pinto (2001, p. 5-6) argumentam sobre a importância da organização deste tipo de pesquisa:

Com os avanços da informática, a seleção de fontes tem podido contar com os bancos de dados existentes, cuja sistematização regular de informação possibilita maior abrangência do levantamento, se, de um lado, eles ampliam o universo contemplado, de outro, armazenam os dados de forma resumida, o que deve ser levado em conta ao se utilizar tais fontes, pois nem sempre os resumos disponibilizam as informações básicas necessárias para a análise. O ideal, nesses casos, seria o exame dos textos originais, ainda que se admita a dificuldade de acesso a eles. Quando não se dispõe de bancos de dados, o próprio processo de elaboração dos estados do conhecimento tem levado à organização desses bancos.

O acesso aos dados em tempo real das Dissertações realizadas na esfera nacional, bem como a extensão territorial do Brasil estão entre os pontos que dificultam esse acesso. $\mathrm{O}$ amplo volume de informações acerca de uma área específica que, com o tempo tem aumentado exponencialmente, tem surtido a necessidade de divulgá-lo para a sociedade, demonstrando por sua vez o importante papel que o EAr desempenha para as pesquisas.

\section{Metodologia utilizada}

Foram determinados parâmetros que serviram de base para a organização desta pesquisa. Por se tratar de uma investigação centrada no Ensino de Geografia, estabeleceu-se o levantamento das Dissertações defendidas, independente do programa ao qual faz parte pa- 
ra que se pudesse ter rápida visualização dos assuntos publicados e consequentemente pesquisados atualmente nestes periódicos. Fez-se necessário, portanto, estabelecer um recorte temporal que desse caráter de atualidade às Dessertações publicadas, decidindo-se por contemplar os últimos cinco anos completos. Logo, o ano de 2013 não entrou na contagem dos dados, sendo, o período compreendido os anos de 2008 a 2012.

Por meio do banco de Teses, no site: http://capesdw.capes.gov.br/capesdw/, que a CAPES mantém, foram escolhidos os seguintes parâmetros de busca:

- Ensino de Geografia;

- Currículo de Geografia;

- Formação de Professor de Geografia;

- Geografia Escolar;

- Educação Especial na Geografia.

A escolha para estes termos de busca se baseou nos itens que mais foram encontrados nos Grupos de Trabalhos (GT) de um determinado Congresso Nacional sobre o Ensino de Geografia, por sua duração e credibilidade, sendo o Encontro Nacional de Práticas de Ensino de Geografia - ENPEG.

Pensando-se na importância de informar os resultados do EAr foram realizadas tabelas, gráficos e mapas. No caso das tabelas, estas mostram as instituições e as Unidades Federativas às quais as pesquisas estão vinculadas. Os gráficos possibilitam a rápida visualização dos conteúdos, a evolução do número de pesquisas defendidas e os assuntos centrais de forma quantitativa. Por fim, os mapas elaborados no software Philcarto ${ }^{1}$, demonstram de modo proporcional a espacialização das produções divididas por Unidade Federativa. A escolha deste programa para a elaboração e confecção dos mapas se deu pelo fato do Philcarto constituir-se um prático software de cartografia temática com facilidade de manusear e manipular os mapas.

\footnotetext{
O Philcarto é um software gratuito desenvolvido pelo geógrafo francês Philippe Waniez. De utilização livre, possibilita a confecção de mapas temáticos, podendo ser obtido por meio de download no site: http://philcarto.free.fr. Os chamados mapas de base cartográfica para o uso neste programa devem estar em formato .ai (Adobe Ilustrator), e as tabelas de informações alfanuméricas em formato Microsoft Excel. Podem-se utilizar os mapas de base cartográfica com outros formatos, como por exemplo: .shp (Shape), por meio do recurso da importação, usando-se, para isso, o Phildigit ou Shape Select. Este software permite confeccionar mapas completos, mapas de círculos proporcionais, mapas de pontos e mapas de fluxo. O programa está disponível em quatro idiomas: francês, inglês, espanhol e português, e como não é um sistema de informações geográficas (SIG), não possui sistema de georreferenciamento. Há duas versões do programa: uma para o Windows e uma para a Macintosh ${ }^{\circledast}$.
} 


\section{Análise dos Dados}

Com o total de 28 programas de Mestrado em Geografia que tiveram ao menos uma Dissertação sobre o Ensino de Geografia publicada em seu respectivo programa entre os anos de 2008 e 2012.

Contudo, o número total de Dissertações neste período, vinculadas aos programas de Geografia foram de 169 pesquisas; conforme Tabela 1, pode-se encontrar Dissertações sobre o Ensino de Geografia em instituições nas seguintes Unidades Federativas: Amazonas, Bahia, Ceará, Distrito Federal, Goiás, Minas Gerais, Mato Grosso do Sul, Mato Grosso, Paraíba, Paraná, Rio de Janeiro, Rio Grande do Norte, Rio Grande do Sul, Rondônia, Santa Catarina, Sergipe e São Paulo, pois bem, das 27 Unidades Federativas, há produções em 17 Unidades.

Tabela 1 - Relação de Instituições de Ensino Superior e Programas de Mestrado em 'Geografia' que Tiveram Alguma Dissertação em Ensino de Geografia Defendida, entre os anos de 2008 e 2012.

\begin{tabular}{|c|c|}
\hline Instituições & UF \\
\hline FUFSE & SE \\
\hline PUCRJ & RJ \\
\hline PUCSP & SP \\
\hline UECE & CE \\
\hline UEL & PR \\
\hline UEM & PR \\
\hline UEPG & PR \\
\hline UERJ & RJ \\
\hline UFAM & $A M$ \\
\hline UFBA & BA \\
\hline UFC & CE \\
\hline UFG & GO \\
\hline UFGD & MS \\
\hline UFMG & MG \\
\hline UFMT & MT \\
\hline UFPB & PB \\
\hline UFPR & PR \\
\hline UFRGS & RS \\
\hline UFRN & $\mathrm{RN}$ \\
\hline UFRO & RO \\
\hline
\end{tabular}




\begin{tabular}{c|c}
\hline Instituições & UF \\
UFS & SE \\
\hline UFSC & SC \\
\hline UFSM & RS \\
\hline UFU & MG \\
\hline UNB & DF \\
\hline UNESP - RIO CLARO & SP \\
\hline UNIOESTE & PR \\
\hline USP & SP \\
\hline
\end{tabular}

Fonte: CAPES, 2013.

Org.: o próprio autor.

Na Tabela 2, verifica-se que em programas de Educação também há Dissertações produzidas para o Ensino de Geografia, isso ocorre muitas vezes, por diversos fatores, contudo vale destacar pelo menos dois: a) a falta de programas Stricto Sensu em Geografia nas instituições, e b) falta de linha de pesquisa própria para o Ensino de Geografia. Ao todo, 53 Dissertações defendidas, entre os anos de 2008 e 2012, entre 35 programas em Educação.

Os Estados, 17 ao todo, que estão representados por alguma instituição são: Alagoas, Ceará, Espírito Santo, Maranhão, Minas Gerais, Mato Grosso, Pará, Paraíba, Piauí, Paraná, Rio de Janeiro, Rio Grande do Norte, Rio Grande do Sul, Rondônia, Santa Catarina, Sergipe e São Paulo.

Tabela 2 - Relação de Instituições de Ensino Superior e Programas de Mestrado em 'Educação' que Tiveram Alguma Dissertação em Ensino de Geografia Defendida, entre os anos de 2008 e 2012.

\begin{tabular}{l|l}
\multicolumn{1}{c|}{ Instituições } & UF \\
\hline CENTRO UNIV. MOURA LACERDA & SP \\
\hline CENTRO UNIV. SALESIANO DE SP & SP \\
\hline FUFPI & PI \\
\hline PUCPR & PR \\
\hline PUCSP & SP \\
\hline UCS & RS \\
\hline UEL & PR \\
\hline UEM & PR \\
\hline UERJ & RJ
\end{tabular}




\section{Instituições}

\section{UF}

UFAL

UFC

$\mathrm{AL}$

\begin{tabular}{|c|c|}
\hline UFC & CE \\
\hline UFES & ES \\
\hline UFF & RJ \\
\hline UFMA & MA \\
\hline UFMG & MG \\
\hline UFMT & MT \\
\hline UFPA & PA \\
\hline UFPB & PB \\
\hline UFPR & PR \\
\hline UFRJ & RJ \\
\hline UFRN & RN \\
\hline UFRO & RO \\
\hline UFS & SE \\
\hline UFSC & SC \\
\hline UNESP - PRESIDENTE PRUDENTE & SP \\
\hline UNISO & SP \\
\hline UNIV. CATÓLICA DE PETRÓPOLIS & RJ \\
\hline UNIVERSIDADE CATÓLICA DE SANTOS & SP \\
\hline UNIVERSIDADE DE PASSO FUNDO & RS \\
\hline UNIVERSIDADE ESTÁCIO DE SÁ & SP \\
\hline UNIV. METODISTA DE PIRACICABA & SP \\
\hline UNIVERSIDADE NOVE DE JULHO & SP \\
\hline UNIVERSIDADE REG. DE BLUMENAU & SC \\
\hline UNOESTE & SP \\
\hline UTP & PR \\
\hline
\end{tabular}

Fonte: CAPES, 2013.

Org.: o próprio autor.

Conforme Tabela 3, Bahia, Minas Gerais, Paraná, Rio de Janeiro, Rio Grande do Sul e São Paulo, apresentam Dissertações defendidas em outros programas que não sejam nem Geografia e nem Educação, muitos extremamente próximos de um ou de outro, mas

a 
nenhum recebe a nomenclatura em questão. Encontrou-se em 23 programas de pós-graduação, nas mais diversas áreas, 35 Dissertações defendidas.

Tabela 3 - Relação de Instituições de Ensino Superior e Programas de Mestrado em ‘Diversos Programas' que Tiveram Alguma Dissertação em Ensino de Geografia Defendida, entre os anos de 2008 e 2012.

\begin{tabular}{l|c|l}
\hline UNESP - PRESIDENTE PRUDENTE & SP & CIÊNCIAS CARTOGRÁFICAS \\
\hline USP & SP & CIÊNCIAS DA COMUNICAÇÃO \\
\hline UFRRJ & RJ & EDUCAÇÃO AGRÍCOLA \\
\hline UNEB & BA & EDU. E CONTEMPORANEIDADE \\
\hline UFRGS & RS & EDU.CIÊNC.QUÍM.VIDA E SAÚDE \\
\hline UNIJUI & RS & EDUCAÇÃO NAS CIÊNCIAS \\
\hline UNESP - BAURU & SP & EDUCAÇÃO PARA A CIÊNCIA \\
\hline UEM & PR & EDU. CIÊNC. E ENS. DE MATEM. \\
\hline UFV & MG & ENGENHARIA CIVIL \\
\hline UFRJ & RJ & ENGENHARIA DE TRANSPORTES \\
\hline UNIVERSIDADE CRUZEIRO DO SUL & SP & ENSINO DE CIÊNCIAS \\
\hline UEL & PR & ENS. DE CIÊNC. E EDUC. MATEM. \\
\hline UNIV. LUTERANA DO BRASIL & RS & ENS. DE CIÊNC. E MATEM. \\
\hline UNICAMP & SP & ENS. E HIST. CIÊNC. DA TERRA \\
\hline
\end{tabular}

Fonte: CAPES, 2013.

Org.: o próprio autor.

Felizmente, os pesquisadores do Stricto Sensu tem tido preocupação com o Ensino de Geografia, o que proporciona uma elevação gradual no decorrer dos últimos anos. Conforme Gráfico 1, num intervalo de apenas cinco anos houve um aumento de quase 37\%.

Espera-se que a tendência seja aumentar a cada ano o interesse em relação a este tipo de pesquisa, objetivando-se assim contribuir com a melhoria do Ensino de Geografia, em todos os níveis de educação. 
Gráfico 1 - Evolução das Dissertações Defendidas em Ensino de Geografia, entre os anos de 2008 e 2012.

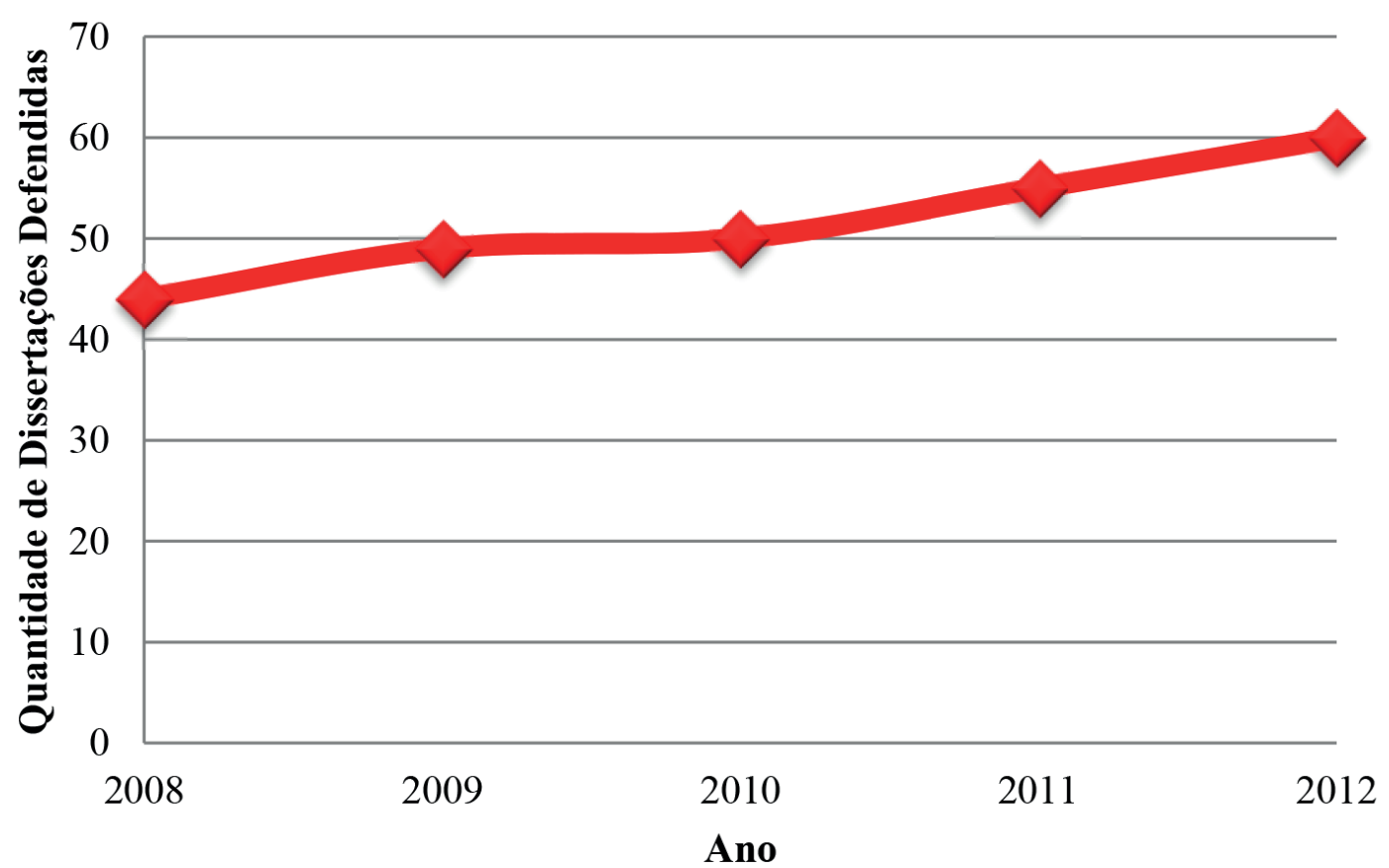

Fonte: CAPES, 2013.

Org.: o próprio autor.

O Gráfico 2 demonstra quais são os programas de Mestrado em Geografia, no Brasil, que mais tiveram Dissertações defendidas sobre o Ensino de Geografia.

A Universidade de São Paulo com 23 pesquisas, seguida pela Universidade Federal de Goiás com 19, logo, mais a Universidade Federal do Rio Grande do Sul com 16 Dissertações defendidas. Entre as que menos tiveram alguma Dissertação defendida: Universidade Federal da Bahia, Universidade Federal do Ceará, Universidade Federal do Mato Grosso, Universidade Estadual de Maringá, Universidade Estadual de Ponta Grossa, Pontifícia Universidade Católica do Rio de Janeiro, Fundação Universidade Federal de Sergipe e Universidade Federal de Sergipe, com apenas uma Dissertação defendida cada. 
Gráfico 2 - Relação de Dissertações Defendidas em Ensino de Geografia, por Instituições, por Programas em Geografia, entre os anos de 2008 e 2012.

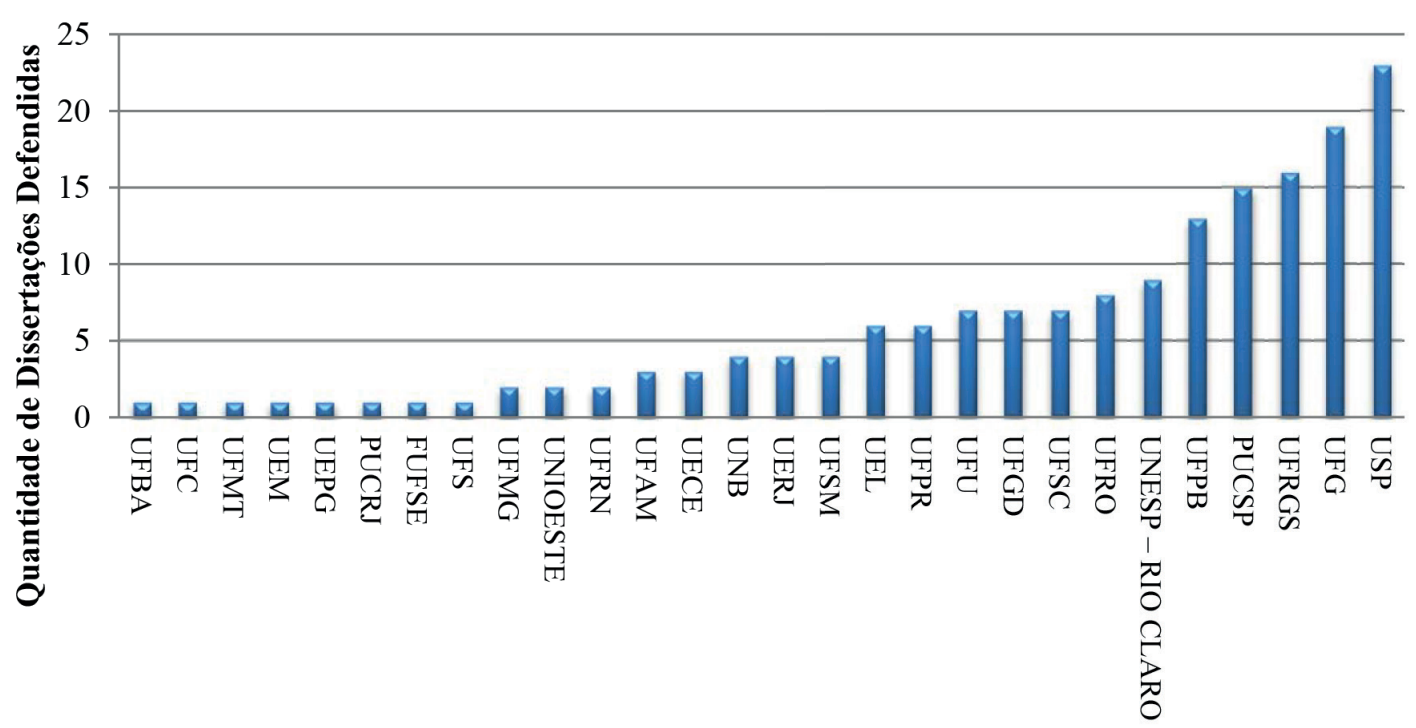

Instituições - Programas de Geografia

Fonte: CAPES, 2013.

Org.: o próprio autor.

Em relação às Dissertações defendidas por meio de programas de Educação, de acordo com o Gráfico 3, nota-se que a Pontifícia Universidade Católica de São Paulo, com cinco pesquisas, e a Universidade Federal do Espírito Santo, com quatro, são as duas instituições que mais defenderam Dissertações em Ensino de Geografia nos programas de Educação. 
Gráfico 3 - Relação de Dissertações Defendidas em Ensino de Geografia, por Instituições, por Programas em Educação, entre os anos de 2008 e 2012.

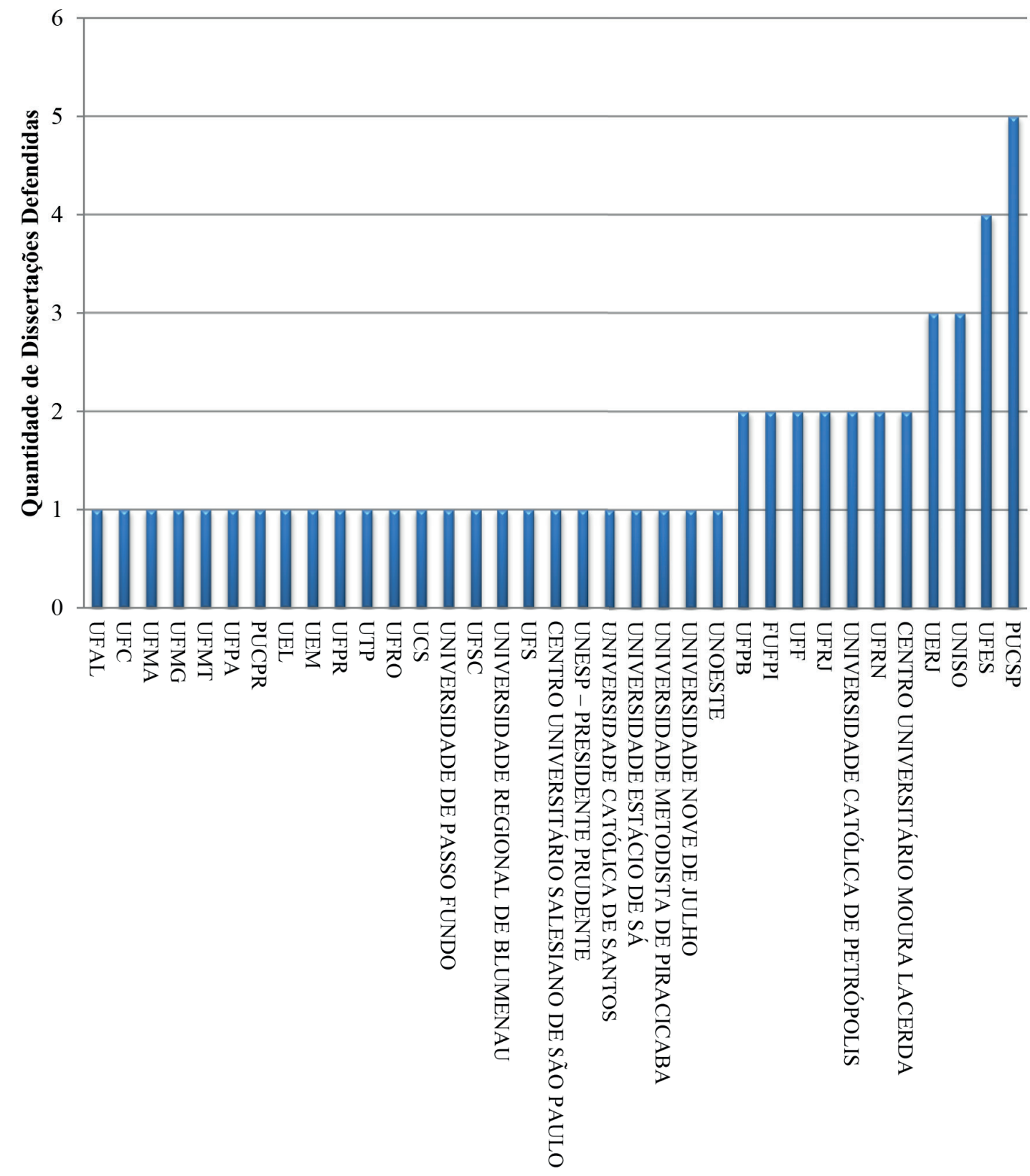

Instituições - Programas deEducação

Fonte: CAPES, 2013.

Org.: o próprio autor.

$\frac{0}{\frac{0}{2}}$ 
A grande maioria de estabelecimentos teve apenas uma Dissertação vinculada ao Stricto Sensu da Educação. (Gráfico 4).

\section{Gráfico 4 - Relação de Dissertações Defendidas em Ensino de Geografia, por Instituições, por Programas Diversos, entre os anos de 2008 e 2012.}

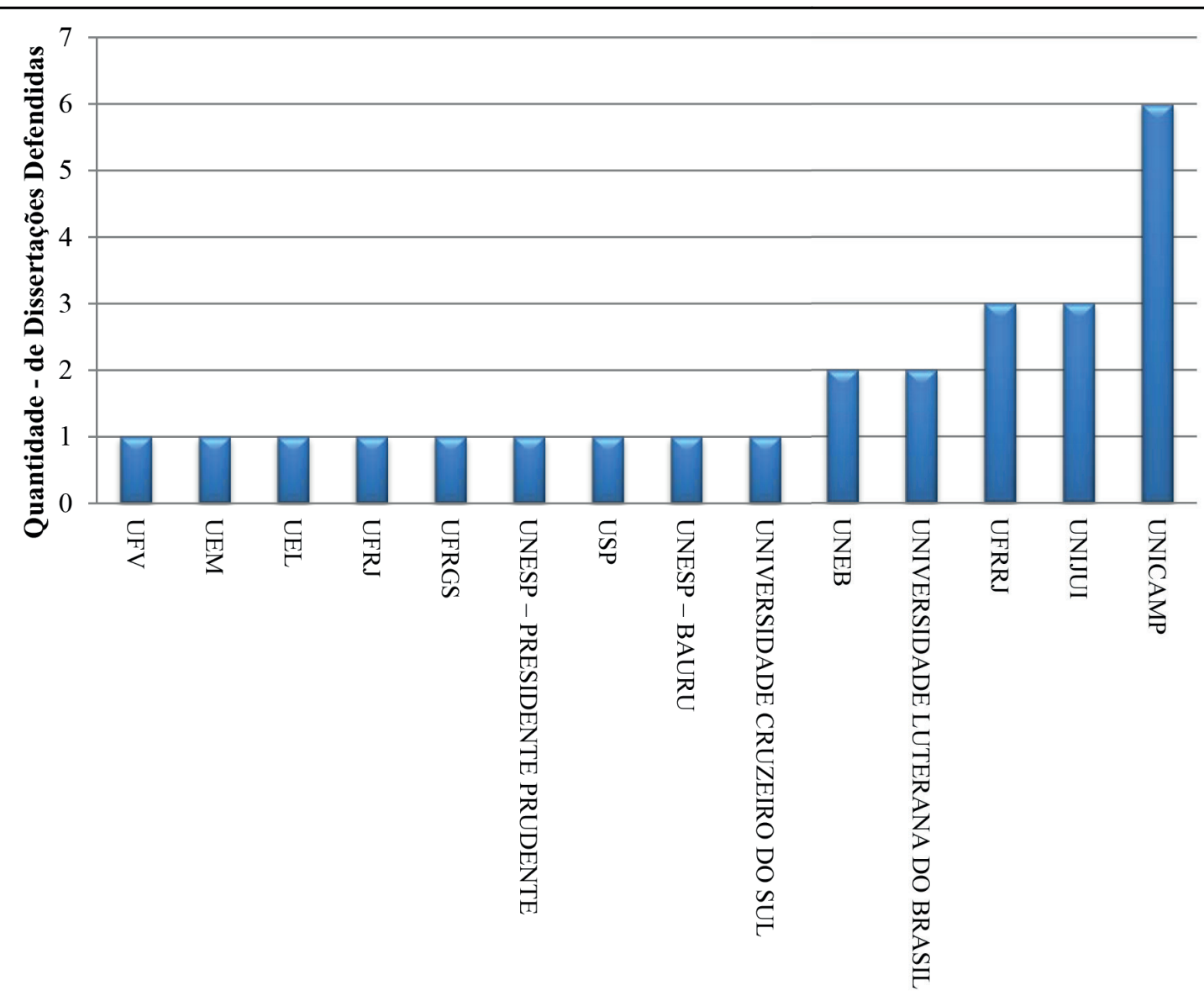

Instituições -Programas Diversos

Fonte: CAPES, 2013

Org.: o próprio autor.

Entre os diversos programas de Stricto Sensu consta a UNICAMP com o programa Ensino e História de Ciências da Terra com seis pesquisas, seguido pelos programas Educação Agrícola e Educação nas Ciências, respectivamente, vinculados a UFRRJ e a UNIJUÍ com três cada. A Universidade Luterana do Brasil e a Universidade Estadual da Bahia aparecem com duas Dissertações defendidas, enquanto as demais instituições apresentaram apenas uma produção, durante os anos de 2008 e 2012.

Em relação ao tipo de instituição, privada ou pública, e, se pública, federal, estadual ou municipal, no Gráfico 5, pode-se observar as devidas proporções. 
Das 86 instituições que proporcionaram as defesas Dissertações de Mestrado, tem-se 63 estabelecimentos públicos, em que 42 são Federais, 20 são Estaduais e uma é Municipal, e foram 23 instituições privadas.

\section{Gráfico 5 - Quantidade de Dissertações Defendidas em Ensino de Geografia, por tipo de Instituição, entre os anos de 2008 e 2012.}

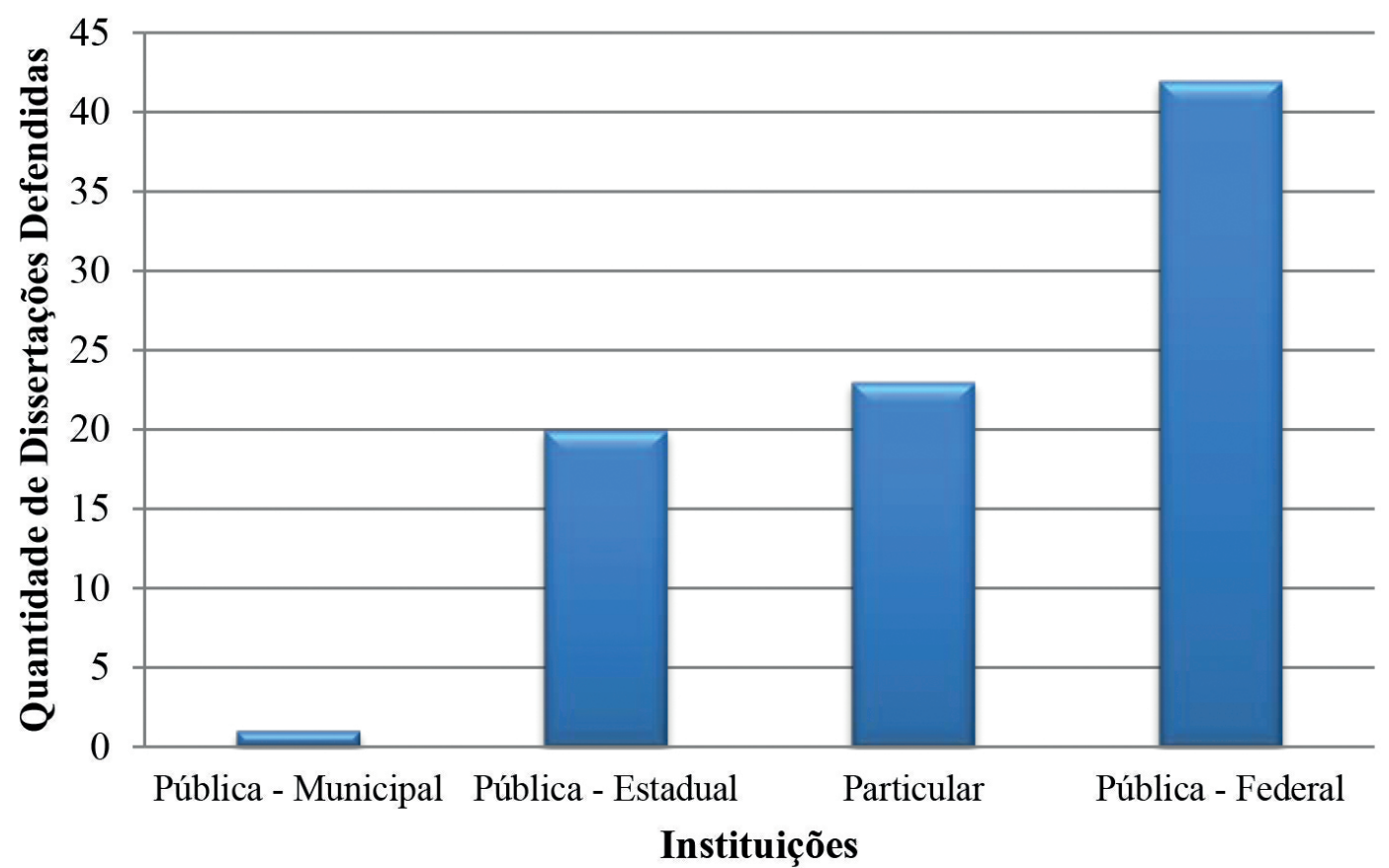

Fonte: CAPES, 2013.

Org.: o próprio autor.

Na Figura 1, consta a distribuição espacial das Dissertações defendidas em território nacional.

Neste mapa pode-se notar que os Estados do Acre, Amapá, Pernambuco, Roraima e Tocantins, não tiveram alguma defesa de Dissertação sobre o Ensino de Geografia, em quaisquer de seus programas.

Contudo São Paulo com 77 Dissertações defendidas está muito a frente do Estado do Rio Grande Sul que, com 29 Dissertações aparece como o segundo Estado com maior quantidade de defesas, em seguida aparece Paraná com 23, Rio de Janeiro com 20, Goiás com 19, Paraíba com 15, Minas Gerais defendeu 14, enquanto Santa Catarina e Rondônia foram nove cada, com sete pesquisas o Estado do Mato Grosso, com cinco segue Ceará, Rio Grande do Norte, Espírito Santo, Distrito Federal e Bahia tiveram quatro defesas, enquanto Sergipe e Amazonas tiveram três, Piauí, Mato Grosso e Alagoas defenderam duas Dissertações e Pará e Maranhão uma produção cada.

a 
Figura 1 - Distribuição de Dissertações Defendidas em Ensino de Geografia, por Unidade Federativa, entre os anos de 2008 e 2012.

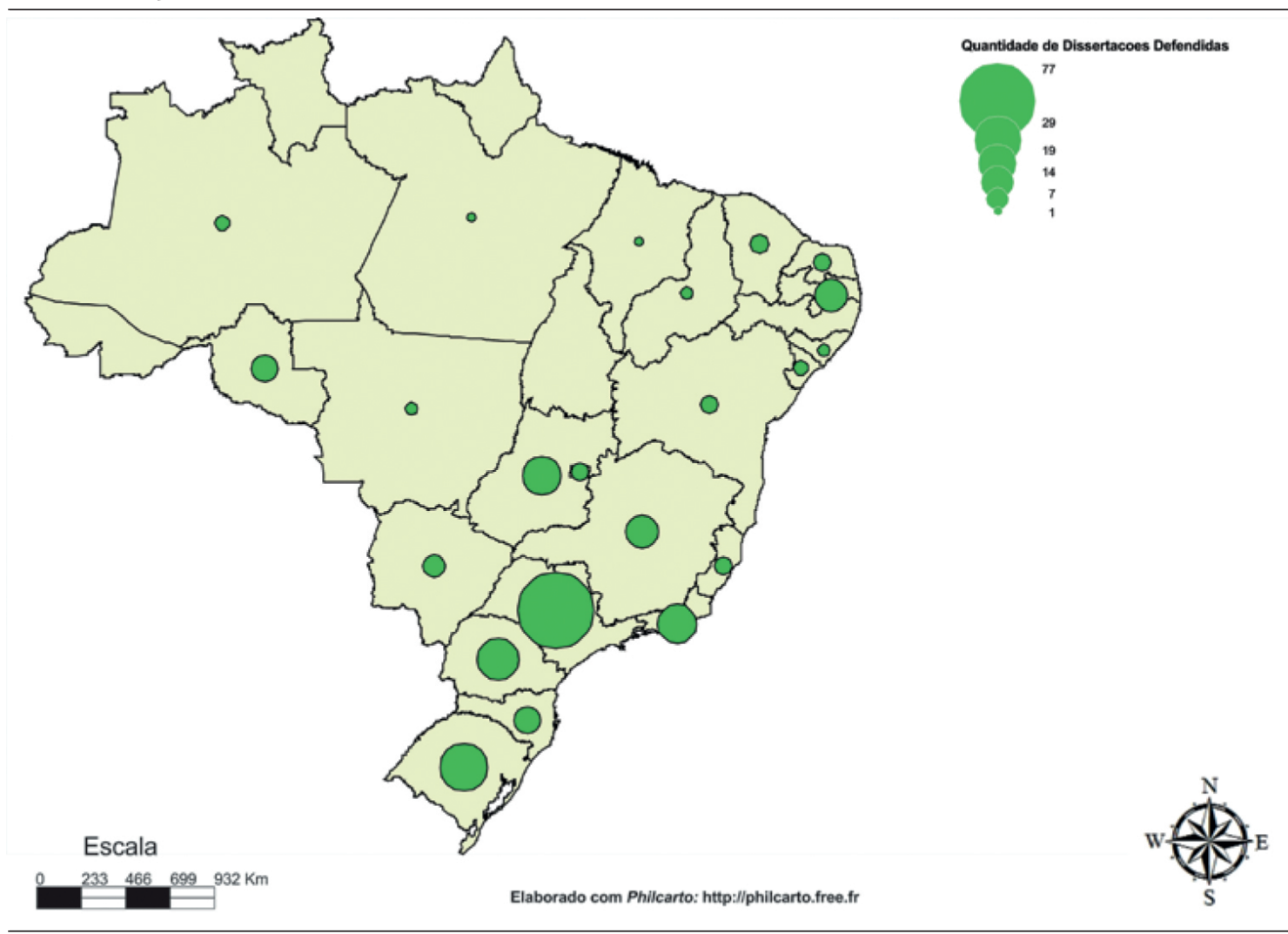

Fonte: CAPES, 2013.

Org.: o próprio autor.

Conforme Figura 2, Distrito Federal, Goiás, Mato Grosso, Mato Grosso do Sul, Espírito Santo, Alagoas, Paraíba, Rio Grande do Norte, Piauí, Maranhão, Pará, Amazonas e Rondônia aparecem com apenas uma instituição que realizou alguma defesa no Stricto Sensu, nível Mestrado.

Santa Catarina, Sergipe e Ceará, possuem dois estabelecimentos de ensino superior que realizaram defesas de Dissertações sobre o Ensino de Geografia. Enquanto Bahia aparece com três, Rio Grande do Sul, Rio de Janeiro e Minas Gerais possuem seis instituições, Paraná conta com sete. O Estado de São Paulo, novamente, aprece como a Unidade Federativa que mais se destaca das demais, neste há 17 instituições que entre os anos de 2008 e 2012 realizaram ao menos uma defesa de Mestrado sobre o tema em questão. 
Figura 2 - Distribuição de Instituições com Dissertações Defendidas em Ensino de Geografia, por Unidade Federativa, entre os anos de 2008 e 2012.

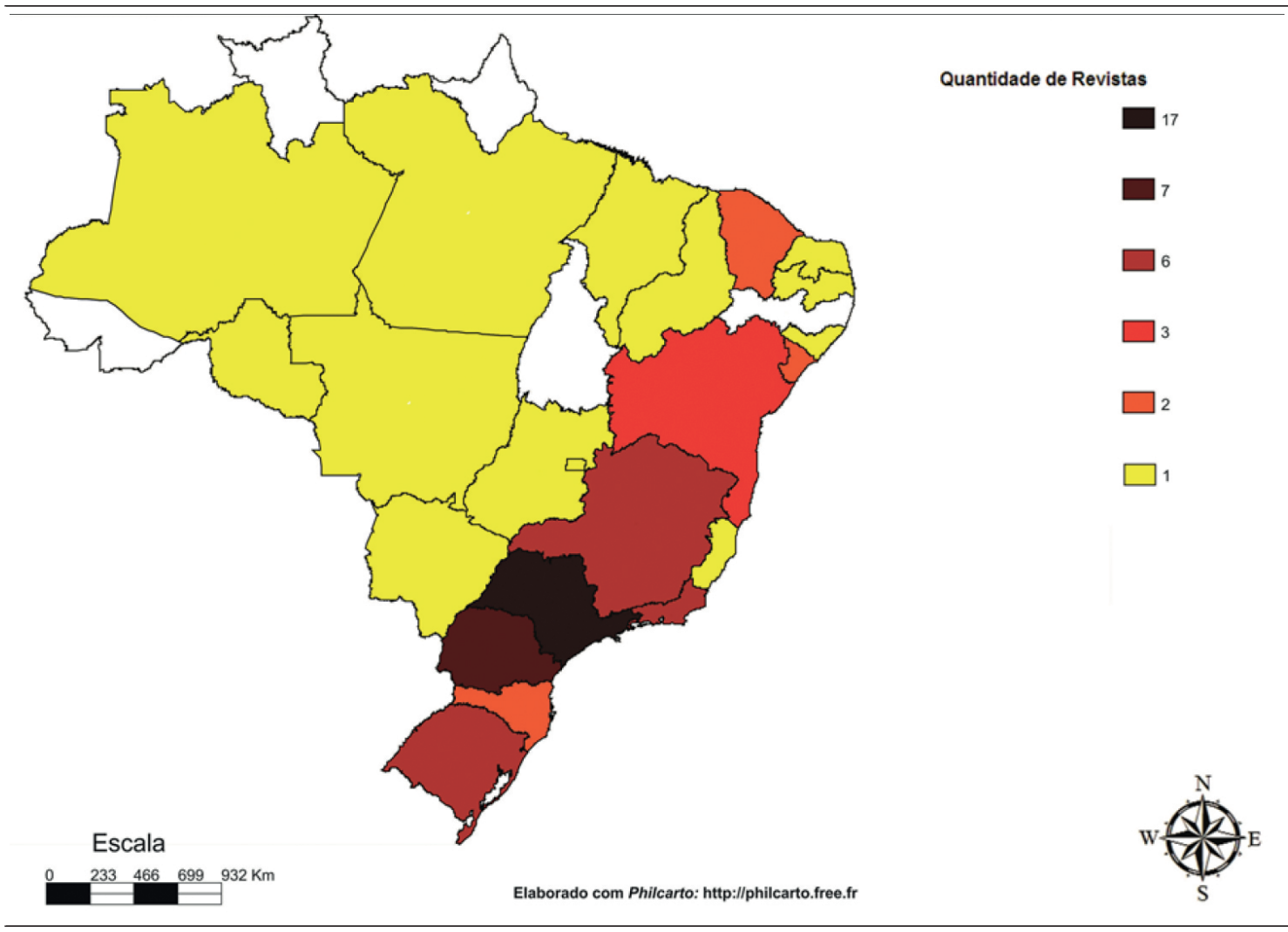

Fonte: CAPES, 2013.

Org.: o próprio autor.

\section{Tematizando as Pesquisas em Ensino de Geografia}

Das 257 Dissertações defendidas, conforme já mencionados anteriormente, muitas destas apresentam dois ou mais assuntos centrais, o que tornou, de certo modo, um pouco complexa a sua organização.

Um fator importante para se levar em consideração está relacionado à forma como foram catalogados os temas de cada Dissertação defendida, que se deu a partir dos títulos para a maioria dessas. Quando não foi possível identificar o assunto dessa maneira, lia-se o resumo e as palavras-chave.

Ao fazer a seleção dos assuntos contidos em cada Dissertação sobre o Ensino de Geografia, observou-se que os temas eram bastante distintos. Organizou-se, portanto, as publicações em torno de seis grandes grupos com temas semelhantes num mesmo setor, como é possível ver Gráfico 6. 
Sobre a Escola: este grupo foi separado das questões que envolvem a modalidade de educação, por conta de que esta última trata sobre os tipos de educação, enquanto neste grupo há reflexões sobre o papel da escola, a organização destas, as formas de avaliação, tipos de escolas e de saberes escolares. Este é menor dos grupos, onde constam 20 Dissertações defendidas sobre o Ensino de Geografia.

Conteúdos Geográficos: neste grupo se encontram as Dissertações que possuem exemplificações de aulas que se remetem a algum conceito específico das aulas de Geografia, têm, também, produções sobre a educação ambiental e epistemologia; neste grupo estão 27 Dissertações defendidas sobre o Ensino de Geografia.

Políticas Públicas: neste terceiro grupo, com 31 Dissertações, abordam sobre o currículo e sua organização.

Modalidade de Educação: a educação, como um todo, possui diversas modalidades de ensino, entre as modalidades que foram encontradas Dissertações sobre o Ensino de Geografia estão a educação do/no campo, educação de jovens e adultos, educação à distância e educação especial, além de debater sobre as séries iniciais e o ensino superior, ao todo este grupo possui 36 Dissertações defendidas.

Sobre o Professor de Geografia: foram 40 defesas relacionadas a formação do professor, seja ela inicial ou continuada, reflexões sobre os aspectos pedagógicos do professor, os saberes docentes e o papel que o professor de Geografia possui frente a sala de aula.

Recursos Didáticos: este grupo, o mais numeroso de todos, com 117 pesquisas defendidas, encontram-se Dissertações sobre a metodologia de ensino, sendo as mais corriqueiras: uso de globos e mapas, novas tecnologias, novas tecnologias e o livro didático. 
O Estado da Arte das Pesquisas em Dissertações de Mestrado em Ensino de Geografia: Contribuição para uma Avaliação a Partir das Pesquisas nos Programas Nacionais de Stricto Sensu (2008 - 2012)

Gráfico 6 - Relação de Temas das Dissertações Defendidas no campo de Ensino de Geografia, entre os anos de 2008 e 2012.

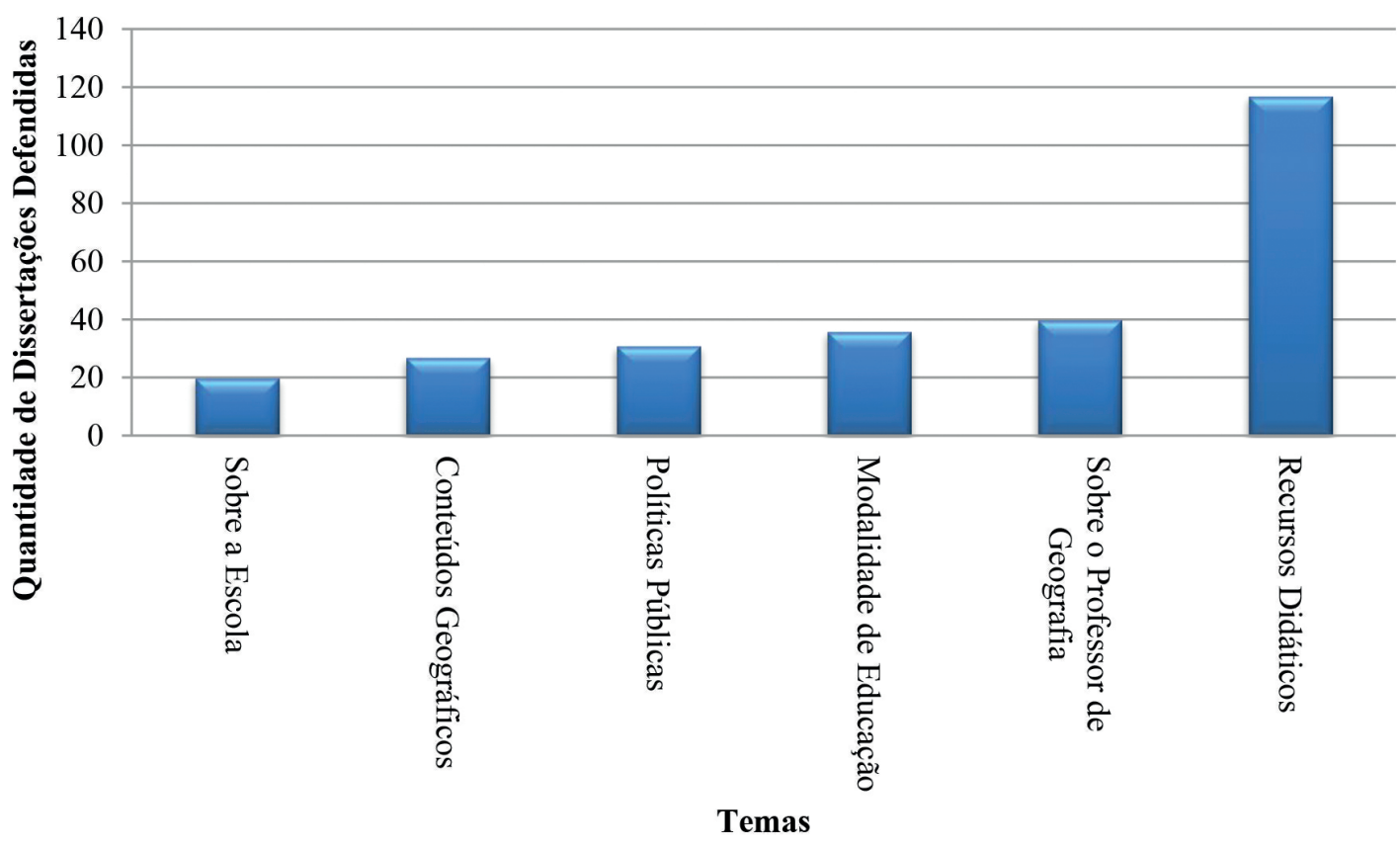

Fonte: CAPES, 2013.

Org.: o próprio autor.

O Gráfico 7 evidencia os assuntos que deram origem aos seis grandes temas discutidos anteriormente. São assuntos que tiveram relevância nas publicações relacionadas ao número de produções ou a originalidade da pesquisa. 


\section{Gráfico 7 - Relação de Assuntos das Dissertações Defendidas em Ensino de Geografia, entre os anos de 2008 e 2012.}

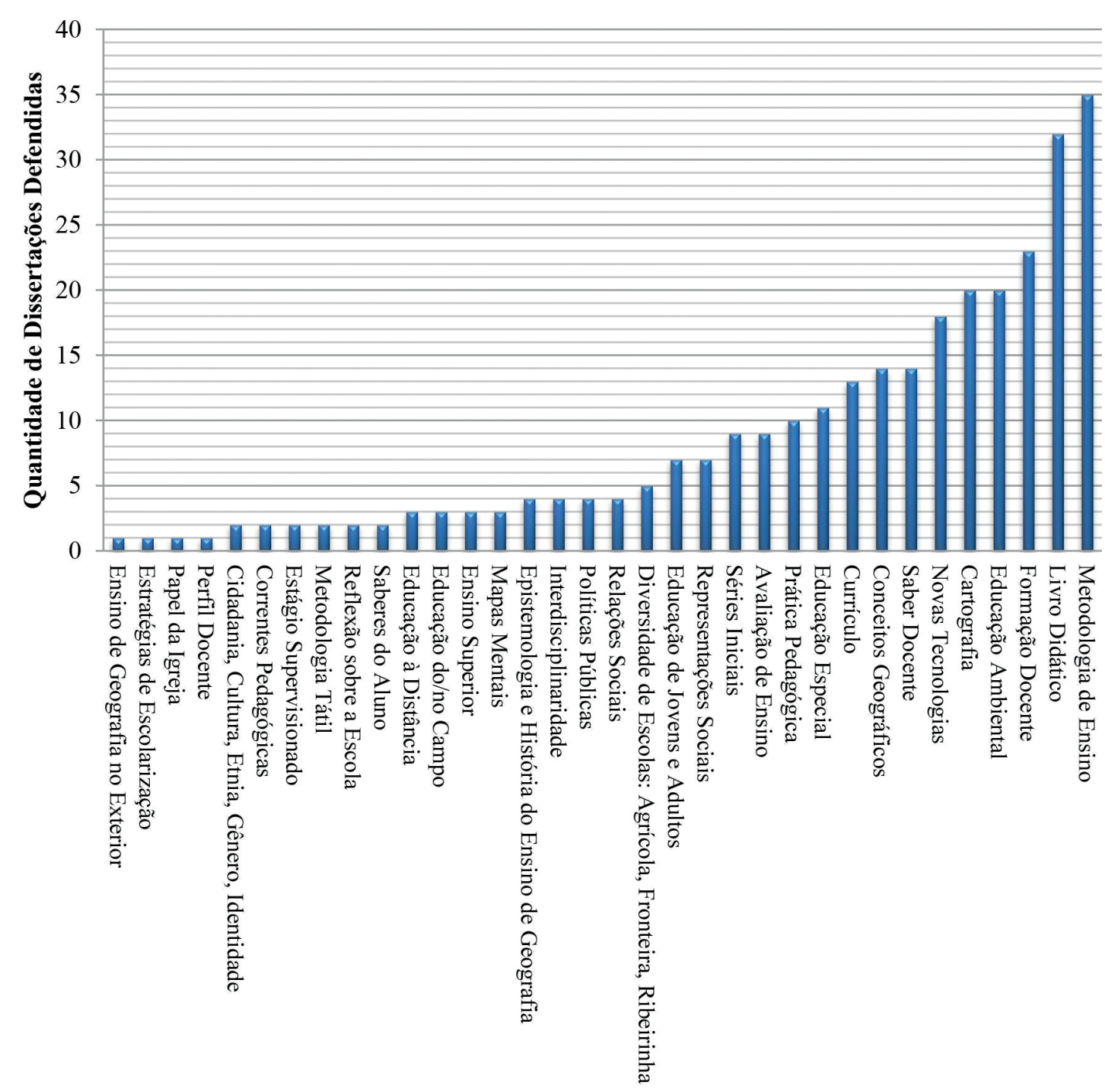

Assunto

Fonte: CAPES, 2013.

Org.: o próprio autor.

\section{Por um Estado da Arte Crítico-Reflexivo}

As pesquisas no campo da Educação estão sustentadas, ao menos, num dos quatro grandes pilares, que são: a Formação Docente, o Ensino, a Aprendizagem e as Questões Curriculares; em que cada um destes pilares possui margem à criação de eixos temáticos que facilitam a catalogação dos temas das pesquisas educacionais. 
Quando se fala em Formação Docente procura-se refletir o papel e o desempenho do educador. Entre os eixos temáticos que fazem parte deste pilar destacam-se: os saberes docentes, a formação inicial e a formação continuada.

Ao se realizar um debate acerca do Ensino deve-se levar em consideração as técnicas e práticas pedagógicas que os educadores utilizam para facilitar a apreensão do conteúdo por parte dos alunos, ou seja, são as metodologias pedagógicas, ao qual cada uma delas corresponde a um eixo temático específico.

Sobre o pilar da Aprendizagem, esta por muitas vezes é entendida, erroneamente, como sinônimo de Ensino. Logo, é necessário esclarecer que trata das teorias educacionais que darão subsídios às concepções dos docentes sobre a Educação. Por exemplo, a Teoria da Aprendizagem Significativa é um eixo temático que se vincula a este pilar. Subsidia os professores a formularem modelos de aulas que auxiliarão na compreensão dos conteúdos por parte dos educandos

Quanto às Questões Curriculares estas podem ser consideradas um pilar complexo e dinâmico, pois nela são encontradas pesquisas sobre modalidades de escola e de educação, currículos, conteúdos, enfim, assuntos relacionados às políticas públicas educacionais.

Poderá ser observada, na Figura 3, a distribuição percentual de Dissertações defendidas sobre o Ensino de Geografia, a partir de levantamento realizado no site da CAPES, entre os anos de 2008 e 2012. Neste diagrama a Aprendizagem aparece em 5\% das pesquisas defendidas, enquanto a Formação Docente está em 16\%, as Questões Curriculares em $38 \%$ e o Ensino aparece em $41 \%$, do total das Dissertações encontradas sobre a temática desta investigação.

Nota-se, então, que as pesquisas estão concentradas entre Ensino e Questões Curriculares (79\%). Enquanto que a Formação Docente e a Aprendizagem possuem pouco mais de um quinto das pesquisas defendidas (21\%). 
Figura 3 - Diagrama Sobre Percentual do Tipo de Pesquisa Educacional em Ensino de Geografia, a partir de levantamento realizado nas das Dissertações Defendidas em Ensino de Geografia, entre os anos de 2008 e 2012.

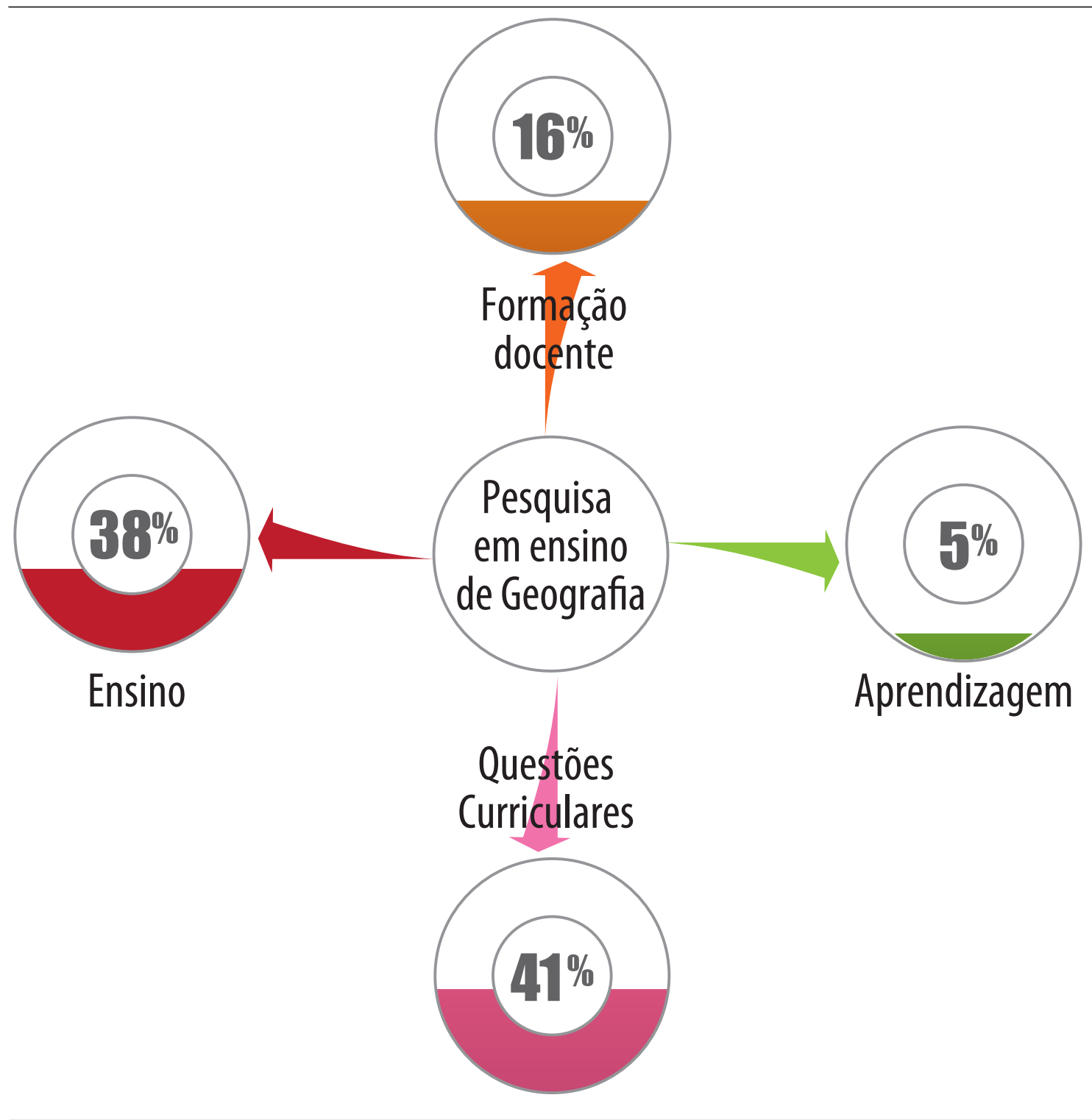

Fonte: CAPES, 2013.

Org.: o próprio autor.

Tão importante quanto a necessidade de não haver uma concentração em apenas um ou dois pilares, é a questão da diversidade de temas pesquisados em cada produção acadêmica.

Os eixos temáticos que formam o pilar do Ensino possuem 120 publicações, 32 destas estão relacionadas aos livros didáticos, 20, aos instrumentos didáticos que auxiliam o ensino de cartografia, enquanto 18 pesquisas sobre o uso das novas tecnologias, e o restante estão entre trabalho de campo, desenhos, fotografias, literatura, mapas mentais e materiais táteis. 
Logo, é importante frisar acerca da necessidade de incentivar o desenvolvimento de pesquisas, desenvolvimento de pesquisas, como, por exemplo, sobre novas tecnologias, uma vez que os alunos estão cada vez mais informatizados. Contudo, é de igual importância que haja variedades em relação aos instrumentos pedagógicos, pois são poucas as variações pesquisadas e há inúmeras outras que podem ser utilizadas em sala de aula para auxiliar os professores no processo de ensino-aprendizagem de Geografia, tais como: atividades lúdicas, charges, tiras e quadrinhos, filmes e vídeos, gráficos, quadros e revistas, maquetes, músicas, e pinturas são exemplos de assuntos que podem e devem ser pesquisados e publicados, com o intuito de fortalecer o pilar do Ensino.

Nas Questões Curriculares, 111 Dissertações defendidas nesta categoria, 34 são referentes as diversas modalidades de educação, 13 são sobre currículo, enquanto os restantes estão divididos entre políticas públicas, conteúdos e estrutura das escolas. Com isso, nota-se que há uma urgente necessidade de pesquisar mais sobre estes dois últimos eixos, objetivando homogeneizar a distribuição de informações que pertencem a este pilar da pesquisa educacional.

Conforme já mencionado, os saberes docentes e a formação inicial e/ou continuada fazem parte do pilar da Formação Docente, que conta com 47 Dissertações defendidas. Nota-se que o número de Dissertações precisa ser aumentar significativamente, de forma a contribuir de maneira crítica e reflexiva com o trabalho do educador.

O pilar da Aprendizagem é o que possui o menor percentual de publicações (5\%). Infere-se que teorias de aprendizagem não estão sendo consideradas pelos pesquisadores para a realização de suas reflexões acerca das produções de Dissertações, enquanto eixo central de suas pesquisas. Deve-se, contudo, estimular o desenvolvimento de pesquisas que mostram o funcionamento das teorias para a aprendizagem dos conteúdos geográficos.

Todos os eixos temáticos possuem sua importância e seu papel dentro do pilar das pesquisas em educação do qual fazem parte, entretanto, igualmente importante é promover pesquisas qualitativas acerca, principalmente, dos eixos que possuem poucas Dissertações defendidas, com o objetivo de fortalecer, de modo homogêneo, todos os pilares da pesquisa em Educação.

\section{Para Não Concluir o Estado da Arte}

Foram 257 pesquisas defendidas entre os anos de 2008 e 2012, por meio de programas de Stricto Sensu, nível Mestrado. Nota-se que a concentração de Dissertações defendidas está nos Estados da região sudeste e sul do país e no Estado da Bahia. 
Isso se dá pelos investimentos em políticas públicas, isto é, são Unidades Federativas que apresentam grande quantidade de programas de Geografia e de Educação, e, consequentemente, recebem mais verbas para financiar as pesquisas, o que gera, por sua vez, uma concentração de pesquisas acadêmicas nessas regiões, favorecendo uma melhor avaliação dos programas, que vem a receber cada vez mais recursos financeiros.

Para que se possa haver maior e melhor distribuição destas pesquisas se faz necessário que os investimentos governamentais tenham critérios mais homogêneos de distribuição para as universidades localizadas em outras regiões do país, dando subsídios um grande empenho das universidades localizadas nas outras regiões do país, para, também, receberem investimentos governamentais, que darão subsídios para o pesquisador realizar sua pesquisa.

Sobre a pesquisa em Ensino de Geografia, deve-se reconhecer a sala de aula como um espaço de reflexões e reivindicações permanente do direito civil e político, da autonomia e da justiça social, pois é importante assumir o compromisso de tornar a geografia escolar um caminho para a construção da cidadania, atribuindo a ela sua real identidade. Por tudo aqui mencionado, até então, é que se faz necessário pesquisar sobre o Ensino de Geografia.

Por fim, pode-se afirmar que, o EAr refere-se a uma atividade árdua e complexa, justamente por seu caráter crítico e reflexivo, auxiliando na otimização e, também, no desenvolvimento de novas demandas de pesquisa, conceitos e paradigmas.

\section{REFERÊNCIAS}

1. BARRETO, E. S. de S.; PINTO, R. P. Avaliação da Educação Básica (1990-1998).

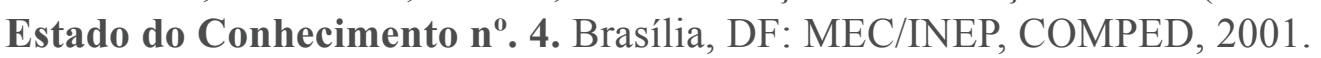

2. CAPES. Banco de Teses. Disponível em: $<$ http://capesdw.capes.gov.br/capesdw/>. Acesso em: 04 junho 2013.

3. FERREIRA, N. S. de A. As pesquisas denominadas "estado da arte". Educação \& Sociedade, Campinas, ano XXIII, nº. 79, p. 257-272, 2002.

4. HADDAD, S. Juventude e escolarização: uma análise da produção de conhecimentos. Estado do Conhecimento $\mathbf{n}^{\mathbf{0}}$. 8. Brasília, DF: MEC/INEP, COMPED, 2002.

5. PHILCARTO. Software Philcarto. Disponível em: $<$ http://philcarto.free.fr $>$. Acesso em: 05 julho 2013. 